\title{
Function Analysis of Mature Products based on Function Unit Table
}

\author{
Tong Yifei ${ }^{1, *}$, Tang Zhaohui ${ }^{1}$, Gu Feng ${ }^{2}$, Zhang Jinlong ${ }^{3}$, Mei Song ${ }^{3}$ and Gong Zhibing ${ }^{4}$ \\ ${ }^{1}$ Nanjing University of Science and Technology, School of Mechanical Engineering 401, 210094 Nanjing, Republic of China \\ ${ }^{2}$ Nantong Vocational University, 226007 Nantong, Republic of China \\ ${ }^{3}$ Nanjing Research Institute for Agricultural Mechanization, Ministry of Agriculture, 210014 Nanjing, Republic of China \\ ${ }^{4}$ Nanjing Kangni New Technology of Mechantronic Company Ltd., 210094 Nanjing, Republic of China
}

Received: 2 Aug. 2013, Revised: 4 Nov. 2013, Accepted: 5 Nov. 2013

Published online: 1 Jul. 2014

\begin{abstract}
Product functional analysis is the foundation of product modularization. Before product module division, function-related analysis of every component or function unit is necessary. As for completely innovative products, the traditional methods are consistent with the habits of designers mind and can help product innovation, while it is difficult to determine the function decomposition granularity. The issue of function decomposition granularity determination challenges the same for mature products. To achieve the function decomposition of made-products, bottom-up function decomposition are proposed and researched. Firstly, determine the possible function unit (bottom function) list for mature products. Secondly, cluster the function units to get their parent functions, and then execute iterated clustering of parent functions until the parent function is product overall function. Finally, products function tree is obtained to achieve function decomposition. In the proposed methodology, the function hierarchy matrix and function incidence matrix are defined to describe the function and the function unit table is built based on which the function units of products are achieved. The relationship between function units achieved above is expressed by using the function hierarchy matrix and function incidence matrix. The function incidence matrix is operated to get the function tree and function decomposition based on bottom-up method by using -intercept. The example shows this method is feasible and easier for programming in computer. Since function units are identified at the beginning, the issue of function decomposition granularity determination is solved.
\end{abstract}

Keywords: Function decomposing, Function hierarchy matrix, Function incidence matrix, Function unit table, Fuzzy clustering

\section{Introduction}

Product functional analysis is the foundation of product modularization. Before product module division, function-related analysis of every component or function unit is necessary first [1]. In 2000, Stone proposed a methodology of quantitative function model for product family design based on function analysis of existing products, which included the following steps: 1)determine sub-functions according to user requirements and build function structure; 2) determine the corresponding module to function structure according to the principles of mainstream, tributaries and converting / transferring; 3) evaluate the satisfaction of product family functions to user function requirements; 4) determine the share module and establish product platforms [2].At present, axiomatic design is a popular method of function decomposition for function analysis [3,4].The general process of function decomposition is to start from production total function and achieve the means this function with astern method until the last function. According to axiomatic design principle, a "functional domains - physical domain - Principles domain" functional decomposition method was proposed, which can convert functions into behavior descriptions [5].As for completely innovative products, the above methods are consistent with the habits of designers mind and can help product innovation, while it is difficult to determine the function decomposition granularity [6].The issue of function decomposition granularity determination challenges the same for mature products.

The present work was carried out in order to solve the product decomposition with the issue of function decomposition granularity determination by discussion and research on bottom-up function decomposition. Firstly, determine the possible function unit (bottom

\footnotetext{
*Corresponding author e-mail: tyf51129@aliyun.com
} 
function) list for mature products. Secondly, cluster the function units to get their parent functions, and then execute iterated clustering of parent functions until the parent function is product overall function. Finally, products function tree is obtained to achieve function decomposition. Since function units are identified at the beginning, the issue of function decomposition granularity determination is solved.

\section{Product function analysis preparation}

\subsection{Product function description}

The essence of product features behaviors as a combination of sub-functions and function units with constraint relations, which generally can be represented by tree structure. In order to analyze product functions, it is necessary to express and quantitatively describe the relationship between sub-functions and function units firstly.

Definition 1: Function tree is denoted as a two-tuple array, $F T=(F, F E)$.

Here, $F T$ denotes a function tree, $F$ denotes function node sets of product function tree and $F E$ denotes edge combinations to describe the subsidiary relationships and constraints among functions. Root node of the function tree represents product function and leaf node represents functions of components. If there aren't leaf nodes or forks somewhere, it means that the leaf node is a function unit, which cannot be decomposed anymore. On the function tree, the leaf nodes are ordered by their importance $I$ and the left leaf is of greater importance than the right at the same layer.

Definition 2: Function hierarchy matrix is an $n \times n$ upper triangular matrix, where $n$ denotes the total number of nodes of function tree, and $n=|F|$.

In function hierarchy matrix, diagonal elements are the function code (may be character) and non-diagonal elements are 0 or 1 .

Set $i, j$ as node of function tree, $(\mathrm{i}, \mathrm{j})$ as the edge connecting node $i$ and $j$, and

$$
(i, j) \in F E=\left\{\begin{array}{l}
0, i \neq j \\
1, i=j
\end{array}\right.
$$

In order to make function tree in accordance with function hierarchy matrix, the importance $I$ is arranged in function hierarchy matrix according to BFS-breadth first search. Here, $I$ denotes the importance of the sub-function at the same layer to the same parent function. Function hierarchy matrix has the following properties:

1) Function hierarchy matrix and function tree are uniquely determined by each other.

2) Diagonal elements of function hierarchy matrix describe the function information of nodes.

3) The order of columns in function hierarchy matrix reflects the importance of nodes at the same layer.
Definition 3: Function incidence matrix reflects the connection relationship and strength between function units, as shown in Figure 1.

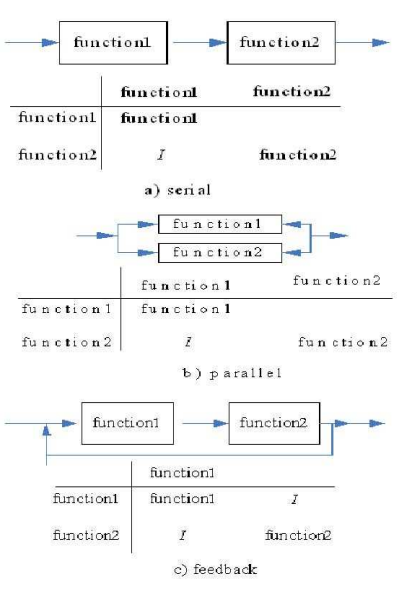

Fig. 1: Function incidence and its expression by function incidence matrix

The functions of one product (indicated by A) can be decomposed as shown in Figure 2(a) as well as the incidence among functions and function units in Figure 2 (b) and 2(c). The function tree of $\mathrm{A}$ product can be transformed to function hierarchy matrix and function incidence matrix as shown in Figure 3.

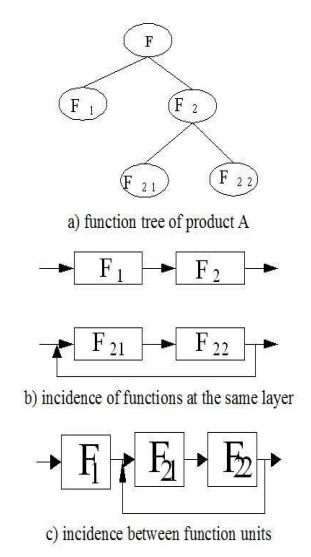

Fig. 2: Product tree and its indication of function incidence

Function incidence matrix reflects the function incidence and incidence strength. In Figure 3, function hierarchy matrix and incidence matrix of product $\mathrm{A}$ are illustrated, where $I_{F_{21}, F_{1}}$ denotes the incidence strength between function $F_{1}$ and function unit $F_{21}$.

2.2 Construction of function incidence matrix 


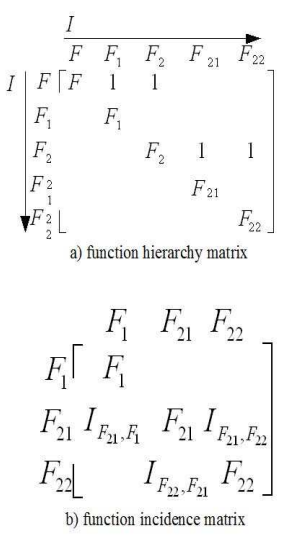

Fig. 3: Function hierarchy matrix and incidence matrix of product A

Since the non-diagonal elements are 0 or 1 and diagonal elements are corresponding function code in function hierarchy matrix, it is easier to get solutions with respect to mathematical computations. While the elements of incidence matrix include incidence strength $I_{i j}, 0$ and corresponding function code, so it is the most important to calculate the incidence strength $I_{i j}$ to construct function incidence matrix.

In literature [7] and [8], the correlations between functions are divided into four types from the view of modular: function-related, assembly-related, space-related and information-related, and the correlation indexes between functions are calculated based on the four types of correlations. In this presented research, the incidence among sun-functions and function units are calculated based the methodology reported in literature [7] and [8].

Four types of correlations between functions [7][8]

(1) function-related: If $f_{i}$ and $f_{j}$ are sub-functions belonging to the same parent function, then $f_{i}$ and $f_{j}$ are function-related;

(2) assembly-related: If there exist assembly correlations between $f_{i}$ and $f_{j}$, then $f_{i}$ and $f_{j}$ are assembly-related;

(3) space-related: If vectors of $f_{i}$ and $f_{j}$ occupy the same space in products, then $f_{i}$ and $f_{j}$ are space-related;

(4) informationCrelated: If there exist the exchange correlations of energy flow, information flow, force flow and so on, then $f_{i}$ and $f_{j}$ are information Crelated.

Suppose the number of correlation types is $n$ and the weight of $k$ th correlation is $W_{k}$, then the incidence between $f_{i}$ and $f_{j}$ can be calculated as:

$$
I_{i j}=\sum_{k=1}^{n} W_{k} r_{i j}^{k}
$$

Here,

$$
r_{i j}^{k}=\left\{\begin{array}{l}
1, f_{i} \text { and } f_{j} \text { meet kth correlation } \\
0, \text { otherwize }
\end{array}\right.
$$

2.3 Function unit table and meta-module table

Definition 4: Function unit refers to the function with no need to decompose anymore in product design.

Definition 5Meta-module refers to the vector of function unit, which can be obtained from 1: n mapping.

Function unit table can be variable due to different product characters and requirements, and function units are coded as well as corresponding meta-modules. A mapping of function unit and meta-modules is constructed as shown in Figure 4. As for mature products, function unit extraction is based on the designers experiences of product structure, and can obtain different extraction results from different views (i.e. design, manufacturing, marketing, transportation, repair and so on). In Figure 4, the meta-modules corresponding to function units are case schemes in essence. The series of meta-module can be obtained by variant design of case schemes [9].

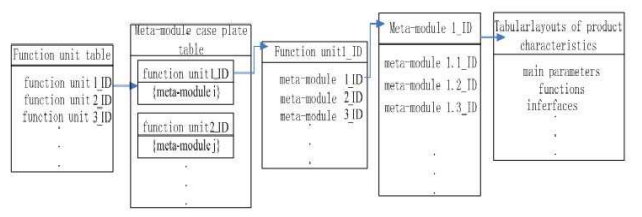

Fig. 4: Mapping of function unit and meta-modules

Function unit extraction is built on mature product structure and the extraction criteria can be summarized as follows from the perspective of function unit vectors [10]:

(1) Function unit should be independent and can be integrated and encapsulated structurally.

(2) Selection, configuration and at most variant design of function unit vectors can be carried out during the procedure of design. Adaptive or innovative designs are not involved.

(3) A function unit can correspond to multiple function unit vectors, while a function unit vector can only to one function unit.

Function unit table collects all the function unit classifications of enterprise and also meta-modules of function unit vectors have the same collection table. A mapping exists between function unit table and meta-module table. 


\section{Function analysis process of made-products}

As for mature products whose parts are determined, function decomposition can be carried out from the structural analysis so as to construct products function tree $[11,12]$. Therefore, product function analysis is actually to construct corresponding product structure tree. In order to facilitate the expression with computers, function hierarchy and incidence matrix discussed above are adopted.

In this presented research, function decomposition for mature products is to construct product function tree based on bottom-up approach and to express using function hierarchy matrix and function incidence matrix defined above.

The basic idea of constructing function tree shown in Figure 5 is as follows:

Step1: Based on analysis of existing products' structure with combinations of function unit table, extracted all the product function units;

Step 2: After analysis of the correlations between function units, calculate the incidence strength between function units and construct function incidence matrix;

Step 3: Obtain the parent functions based on based on fuzzy clustering tree with multi- $\lambda$ for function units clustering;

Step 4: Repeat step 3 until the superior function become product overall functionality.

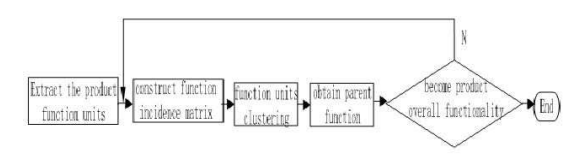

Fig. 5: Construction procedure of function tree

\section{Case study}

After structure analysis of one product, function units $F_{1}-F_{9}$ are obtained, whose incidences are illustrated in Figure 6(a). The incidence strength can be calculated using the above method to construct function incidence matrix. Fuzzy clustering tree with multi- $\lambda$ is adapted to convert the function incidence matrix $[7,8]$ (Here, $\lambda=0.7$ ) . Then the superior functions F'1-F' 3 of function units are obtained, whose incidence are illustrated in Figure 6(b), and the function incidence matrix can be stripped out of the matrix in Figure 6(a). The stripped procedures are as follows:

(1) Determine the function unit pair sets, incidence strength of which is less than $\lambda$ in Figure 6(a), and the incidence strength of each function unit pair. Express them as $f_{i}$ and $f_{j}$, where $f_{i} f_{j}$ and $I_{i j}^{\prime}$ respectively function unit $i$, function unit $j$ and their function incidence strength.

(2) Search the parent function (F'1-F'3) corresponding to each function unit pair.

(3) The incidence strength of function unit pair $I_{m n}^{\prime}$, and

$$
I_{m n}^{\prime}=\sum^{N} I_{i j}
$$

where $I_{m n}^{\prime}$ denotes the incidence between $F_{n}$ and $F_{m}$

$$
\left(F_{n}, F_{m} \in\left\{F_{1}^{\prime}, F_{2}^{\prime}, F_{3}^{\prime}\right\}, f_{i} \in F_{n}, f_{j} \in F_{m}\right),
$$

and $N$ denotes the number of function unit pairs.

(4) Calculate and construct the new function incidence matrix. If the incidence matrix in Figure 6(b) is converted further with $\lambda=0.5$, then F' 1 and F' 2 can be clustered to F"1 with high superior. The final function tree can be obtained as shown in Figure 6(c) after ordering the functions according to their importance, which can be calculated based on the sensitivity analysis and calculation of corresponding structures.

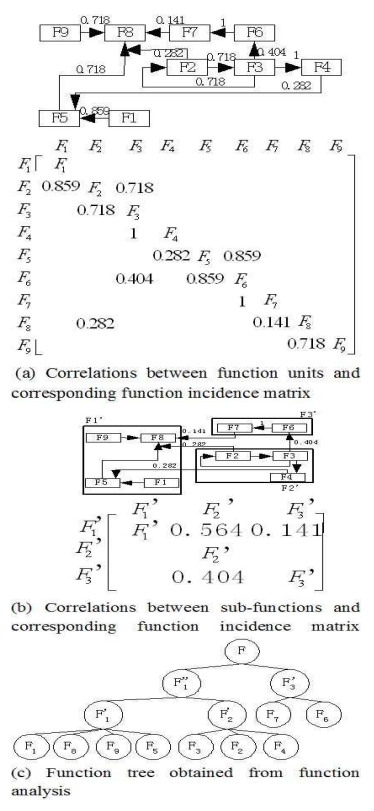

Fig. 6: Case study procedure of function analysis 


\section{Conclusions}

Functional analysis is essential regardless of product innovative design or variant design. The main results of this research can be concluded as follows:

1) Functional analysis of mature products is discussed in detail. It is favourable for computer programming and solution to express functions and their incidence with function hierarchy matrices and function incidence matrix.

2) Function unit tables are established, based on which too coarse or too small granularity of function decomposition can be avoided.

3) The corresponding of function unit table to meta-module table is also discussed. With combinations of function decomposition and the function-structure mapping, the research can lay good foundations for mature product modularization.

\section{Acknowledgement}

This work was financially supported by the National Natural Science Foundation of China (61104171), Postdoctoral Program of Science Foundation of Jiangsu Province of China (0901041C), the Fundamental Research Funds for the Central Universities and Zijin Star of Outstanding Program of NJUST. The supports are gratefully acknowledged. The authors would also acknowledge the Editor of Applied Mathematics \& Information Sciences.

\section{References}

[1] Mehdi Iraqi, Lionel Roucoules. From Functional Analysis to CAD Modeling Based on Knowledge Transformation Driven by the Design Process, IFIP Advances in Information and Communication Technology, 388, 458-467 (2012).

[2] Robert B.Stone. Using quantitative functional models to develop product architecture, Design Studies, 21, 239-260 (2000).

[3] Jeonghun Ahn, Sung Woon Cha. Continuous functional analysis for axiomatic design, International Journal of Precision Engineering and Manufacturing, 12, 153-156 (2011).

[4] Osman Kulak, Selcuk Cebi, Cengiz Kahraman. Applications of axiomatic design principles: A literature review, Expert Systems with Applications, 37, 6705-6717 (2010).

[5] SUN N P. Design Axioms and Quality Control, Robotics and Computer Integrated Mannufacturing, (1992).

[6] Cai Chi-lan, Bai Yue-wei, Xia Yan-chun, Wang Xiaogang, Liu Kai. A Function Analysis Model Based on Granular Computing and Practical Example in Product Data Management, Physics Procedia, 24, 1140-1146 (2012).

[7] HOU Liang. The theory, method and practice application of modular design for mechanical product, dissertation, Zhejiang University.
[8] JIANG Hui, XU Yanshen, XIE Yan. Research on Modular Design for Mechanical Product, Manufacturing Technology \& Machine Tool, 7-9 (1999).

[9] Lippmann, R.P., An introduction to computing with neural nets, ASSP Magazine, IEEE, 4, 4-22 (1987).

[10] Fei Yu,Long Fei Wang,Run Hua Tan, Hui Jin. An improved functional decomposition method based on FAST and the method of removal and operation, System Science and Engineering (ICSSE), 487-492 (2012).

[11] Sukanesh R, Harikumar R. Fuzzy techniques and hierarchical aggregation functions decision trees for the classification of epilepsy risk levels from EEG signals, TENCON-IEEE, 6-7 (2008).

[12] Dongmin Zhang, Dachao Hu, Yuchun Xu, Hong Zhang. A framework for design knowledge management and reuse for Product-Service Systems in construction machinery industry, Computers in Industry, 63, 328-337 (2012).

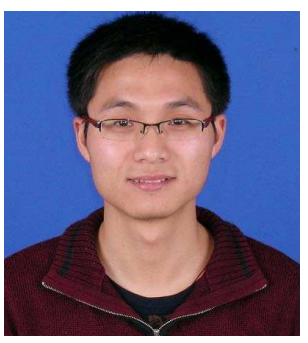

Tong Yifei received the $\mathrm{PhD}$ degree in Mechanical Engineering Science and now is an associate professor at Nanjing University of Science and Technology.His research interests are in the areas of modern product development and advanced manufacturing system management and control.

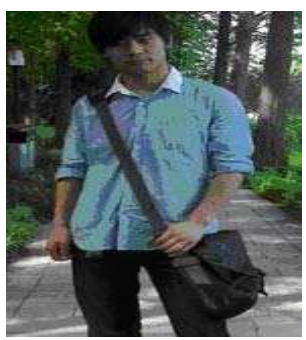

Tang Zhaohui is
a master $r$ student
in Mechanical Engineering
Science.His r research
interest is energy-saving
product development.

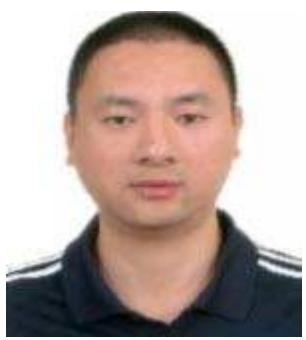

Gu Feng received the Master degree in Mechanical Engineering Science at Nanjing University of Science and now is a lecture at Nantong Vocational University of Science.His research interest is mechanical system design and control.He has published research articles in several 

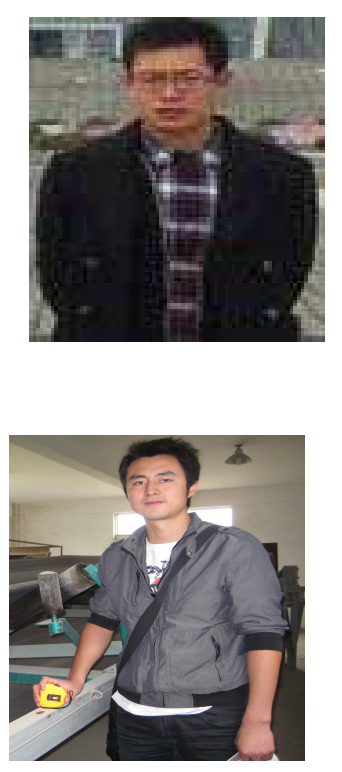

Zhang Jinlong is an Research Assistant at Nanjing Research Institute for Agricultural Mechanization Ministry of Agriculture.

Mei Song received the Master degree in Mechanical Engineering Science at Nanjing University of Science and now is an Research Assistant at Nanjing Research Institute for Agricultural Mechanization Ministry of Agriculture.His research interest is Agricultural engineering machinery.

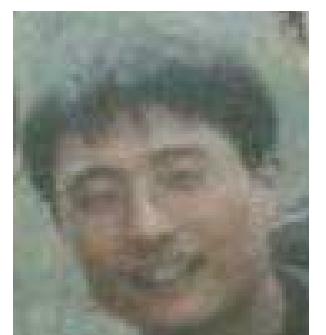

Gong Zhibing received the $\mathrm{PhD}$ degree in Mechanical Engineering Science at Nanjing University of Science and Technology.His research interest is urban rail transit system design and management. 